\title{
Downregulation of ubiquitin E3 ligase TNF receptor-associated factor 7 leads to stabilization of p53 in breast cancer
}

\author{
LIXIN WANG $^{1 *}$, LEI WANG $^{1 *}$, SHUPING ZHANG ${ }^{1}$, GUANGBO QU $^{1}$, \\ DAOQIANG ZHANG ${ }^{1}$, SHITAO LI ${ }^{2}$ and SIJIN LIU ${ }^{1}$ \\ ${ }^{1}$ State Key Laboratory of Environmental Chemistry and Ecotoxicology, Research Center for \\ Eco-Environmental Sciences, Chinese Academy of Sciences, Beijing 100085, P.R. China; ${ }^{2}$ Division of Immunology, \\ Department of Microbiology and Immunobiology, Harvard Medical School, Boston, MA 02115, USA
}

Received August 31, 2012; Accepted September 28, 2012

DOI: $10.3892 /$ or.2012.2121

\begin{abstract}
. p53 is a key tumor suppressor and a master regulator of various signaling pathways, such as those related to apoptosis, cell cycle and DNA repair. In this study, we found a pronounced cytosolic accumulation of the 553 protein in a panel of breast cancer specimens. Several mutations lead to p53 accumulation by disruption of MDM2-mediated p53 degradation. However, gene sequencing revealed no p53 mutation in the majority of our samples. Through search for other possible p53 E3 ligases by mRNA and protein expression analysis, downregulation of TNF receptor-associated factor 7 (TRAF7) expression was found in these breast tumors. We further identified TRAF7 as an E3 ligase for K48-linked ubiquitination of p53 in vitro. These results suggested that the p53 accumulation was due to the defects of TRAF7-mediated ubiquitination. The downregulation of $T R A F 7$ also correlated with poor prognosis in a breast cancer cohort. Collectively, TRAF7-mediated ubiquitination of p53 plays a critical role in breast cancer development, and these insights may aid in the development of novel therapeutic strategies for breast cancer.
\end{abstract}

\section{Introduction}

In normal cells, the wild-type (wt) p53 protein is rapidly degraded by the proteasome and therefore has a short half-

Correspondence to: Professor Sijin Liu, State Key Laboratory of Environmental Chemistry and Ecotoxicology, Research Center for Eco-Environmental Sciences, Chinese Academy of Sciences, 18 Shuangqing Road, Beijing 100085, P.R. China

E-mail: sjliu@rcees.ac.cn

Dr Shitao Li, Division of Immunology, Department of Microbiology and Immunobiology, Harvard Medical School, Boston, MA 02115, USA

E-mail: shitao_li@hms.harvard.edu

*Contributed equally

Key words: breast cancer, TNF receptor-associated factor 7, p53, cytosolic retention, ubiquitination life. MDM2 is a p53-induced ubiquitin E3 ligase, which in turn targets p53 for degradation. When mutant p53 loses its trans-activating function, it cannot induce $M D M 2$, leading to the accumulation of p53. However, the accumulation of wt p53 has also been observed in some human tumors. The tumor suppressor protein, p53, is encoded by the TP53 in humans. Its fundamental function is to guard the integrity of the genome, and thus prevent cancer initiation. p53 exerts an anticancer function through a number of mechanisms including its significant role in apoptosis, DNA repair and cell cycle regulation. In normal cells, the $\mathrm{p} 53$ protein is maintained at a rather low level via a continuous degradation. On the one hand, the binding of MDM2 to p53 prohibits its transcriptional activities and enhances its transportation from nucleus into cytoplasm; on the other hand, MDM2, as an E3 ubiquitin ligase, covalently ubiquitinates p53 and subjects p53 for degradation by proteasomes. Approximately 50\% of human tumors have an increased concentration of the p53 protein (1). The increased p53 level is due to genetic mutations in most cases; meanwhile, p53 accumulation often remains in wt in the other cases. The impairment of p53 degradation and its functional deficiency might account for the accumulation of wt p53 in cancer cells. Nonetheless, the abundance of wt p53 is sufficiently compensated and well tolerated in cancer cells.

TNF receptor-associated factor 7 (TRAF7) is an E3 ubiquitin ligase for several proteins involved in the $\mathrm{NF}-\kappa \mathrm{B}$ pathway, such as c-FLIP (2), NF- $\mathrm{B}$ essential modulator (NEMO) and the p65 subunit of NF- $\kappa \mathrm{B}$ transcription factor $(3,4)$. Here, we demonstrated that TARF7 was an E3 ligase for p53 and reduction of TRAF7 led to cytosolic accumulation of p53. In the breast tumor specimens enlisted in the present study, pronounced p53 accumulation was observed, particularly in cytoplasm; however, gene sequencing analysis suggested an extremely low frequency of mutation in the $p 53$ gene in these tumors. Meanwhile, largely downregulated TRAF7 expression was found in these tumors, correlating with the high levels of p53. TRAF7 downregulation therefore might, at least partially, explain the mechanism responsible for wt p53 accumulation in breast tumors. Additionally, TRAF7 downregulation indicated poor prognosis in a breast cancer cohort, particularly with concomitant upregulation of $p 53$. These data collectively suggested that TRAF7 plays an important role in stabilizing 
p53, and TRAF7 deregulation results in defects in the p53 degradation pathway. The deregulation of the TRAF7/p53 axis likely contributes to the development of breast cancer.

\section{Materials and methods}

Breast tumor specimens. All tumor specimens from breast cancer patients were obtained from the Wendeng Central Hospital, Shandong, China. The analyses of the human tumor specimens were conducted according to the guidelines of the ethics committee at the Wendeng Central Hospital, and were also approved by the ethics committee at RCEES, Chinese Academy of Sciences.

Immunohistochemical assay. The p53 monoclonal antibody DO-7 was purchased from Santa Cruz Biotechnology. Evaluation of the immunohistochemical analysis was conducted blindly without knowledge of the clinical and pathological characteristics of the patients. The intensity of p53 staining was quantified with the software Image-Pro Plus.

Mutation spectrum analysis of the p53 gene. Breast tumor samples were analyzed for DNA sequence mutations within the exons 4-9 of the $p 53$ gene as previously described $(5,6)$. Briefly, DNA was isolated from tumor tissues with QIAamp DNA Micro kit (Qiagen). PCR products were purified with QIAquick Gel Extraction kit (Qiagen) and sequenced in Sangon Biotech Co., Ltd. (Shanghai, China). Primers for PCR amplification are listed in Table I.

Ubiquitination assay in vitro. In vitro ubiquitination assays were performed according to the manufacturer's manual (Boston Biochem). E1, UbcH5c, ubiquitin and reaction buffer were purchased from Boston Biochem. Recombinant p53 was purchased from BD Pharmingen. TRAF7 and TRAF7 mutant (with RING domain deletion) were purified from 293 cells expressing FLAG-TRAF7 or mutant.

$q R T-P C R$ analysis. qRT-PCR analysis was performed as previously described (7). Primer sequences for human TRAF7 in PCR analysis were: forward, 5'-GTGTGGTGTCTCTGCG TCTAC-3'; reverse, 5'-ACACAATGATGGTGCAGTCTG-3'. GAPDH was used as the internal control. The primer sequences for GAPDH were: forward, 5'-GAAGGTGAAGGTCGGA GT-3'; reverse, 5'-GAAGATGGTGATGGGATTTC-3'.

Western blot analysis. Western blot analysis for TRAF7 was performed as previously described (8). The intensities of TRAF7 bands were quantified with ImageJ and normalized to GAPDH. Anti-GAPDH and anti-TRAF7 antibodies were purchased from Santa Cruz Biotechnology and Abcam, respectively.

Survival analysis in a breast cancer cohort. A cohort dataset was used to assess the correlation between the altered expression of TRAF7 and $p 53$ and the prognosis in breast cancer patients. The dataset was generated using Illumina HumanHT-12 v3 Expression BeadChips from 183 primary breast cancer samples. The normalized mRNA expression data were obtained from the GEO database (GEO ID: GSE 24450)
Table I. Primers used in the current study for exons 4-9 of the p53 gene.

\begin{tabular}{|c|c|}
\hline Location & Primer sequence $\left(5^{\prime}-3^{\prime}\right)$ \\
\hline Exon 4 & $\begin{array}{l}\text { Forward: cctggtcctctgactgctcttttcaccca } \\
\text { Reverse: ggccaggcattgaagtctcat }\end{array}$ \\
\hline Exon 5 & $\begin{array}{l}\text { Forward: atctgttcacttgtgccetgactttc } \\
\text { Reverse: accctgggcaaccagccctgtc }\end{array}$ \\
\hline Exon 6 & $\begin{array}{l}\text { Forward: agagacgacagggctggttg } \\
\text { Reverse: cttaacccctcctcccagag }\end{array}$ \\
\hline Exon 7 & $\begin{array}{l}\text { Forward: ctcctaggttggctctg } \\
\text { Reverse: gaggctggggcacagcaggccagtg }\end{array}$ \\
\hline Exon 8 & $\begin{array}{l}\text { Forward: ccttactgcctcttgcttct } \\
\text { Reverse: ataactgcacccttggtctc }\end{array}$ \\
\hline Exon 9 & $\begin{array}{l}\text { Forward: ggagaccaagggtgcagttatgcctcag } \\
\text { Reverse: cccaattgcaggtaaaacag }\end{array}$ \\
\hline
\end{tabular}

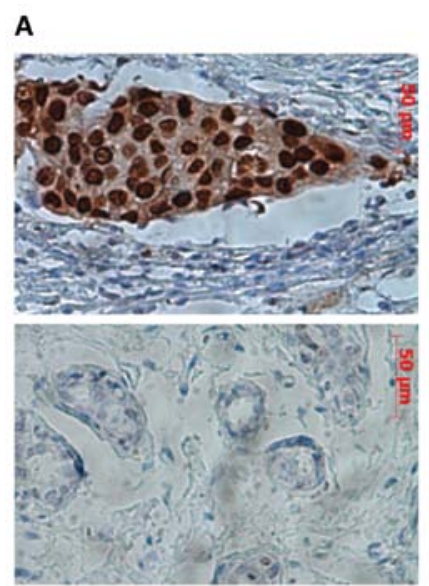

B

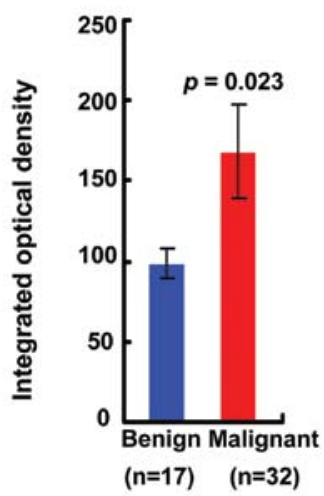

Figure 1. Immunohistochemical staining of p53 in breast tumor specimens (A) Representative images of p53 staining in breast cancer specimens with the positive staining in the upper panel and the negative staining in the lower panel. (B) The quantified data of the integrated optical density for p53 staining in malignant tumors $(n=17)$ compared to the benign tumors $(n=32)$.

(9). The mean value of $p 53$ and TRAF7 expression were used as threshold to determine expression levels. Overall survival was analyzed by the method of Kaplan-Meier plot.

Statistical analysis. The two-tailed Student's t-test was used to analyze experimental data. Data are shown as the means \pm SE. $\mathrm{P}<0.05$ was considered to indicate statistically significant differences.

\section{Results}

Accumulation of p53 in breast tumors. p53 protein levels were examined in 30 benign and 36 malignant tumors using immunohistochemical assays. Three distinct staining levels (negative, weak positive and positive) were characterized according to the staining intensity (Fig. 1A). In total, 30\% (9 out of 30) of the benign tumors were p53 negative, while 
Table II. Immunohistochemical analysis of p53 in breast cancer specimens.

\begin{tabular}{lcc}
\hline Pattern & $\begin{array}{c}\text { Benign } \\
\text { tumors }\end{array}$ & $\begin{array}{c}\text { Malignant } \\
\text { tumors }\end{array}$ \\
\hline Negative & $30 \%(9 / 30)$ & $8 \%(3 / 36)$ \\
Weak positive & $13 \%(4 / 30)$ & $3 \%(1 / 36)$ \\
Positive & $57 \%(17 / 30)$ & $89 \%(32 / 36)$ \\
Grade of cytosolic staining & ++ & +++ \\
Grade of nuclear staining & + & + \\
\hline
\end{tabular}

Table III. Sequencing results of p53 genomic DNA from tumors.

\begin{tabular}{lcccc}
\hline & $\begin{array}{c}\text { No. of } \\
\text { patients }\end{array}$ & $\begin{array}{c}\text { No. of } \\
\text { mutation }\end{array}$ & $\begin{array}{c}\text { Mutation } \\
\text { type }\end{array}$ & Frequency \\
\hline Exon 4 & 98 & $1^{\text {a }}$ & $\mathrm{A} \rightarrow \mathrm{G}($ Val $\rightarrow$ Ala $)$ & $1.02 \%$ \\
Exon 5 & 102 & 0 & & 0 \\
Exon 6 & 104 & 0 & & 0 \\
Exon 7 & 101 & 0 & & 0 \\
Exon 8 & 99 & 0 & & 0 \\
Exon 9 & 97 & 0 & & 0 \\
\hline
\end{tabular}

The mutation was located at codon 103 in exon 4.

only $8 \%$ ( 3 out of 36 ) of the malignant tumors were negative (Table II). By contrast, $89 \%$ of the malignant tumors (32 out of 36) showed p53 positive while $57 \%$ of the benign tumors (17 out of 30) were p53 positive (Table II). There was a significant increase of the integrated optical density for the p53 staining in malignant tumors when compared to the benign tumors $(\mathrm{P}<0.05)$ (Fig. 1B). Moreover, we observed more pronounced cytoplasmic retention of p53 in malignant tumors than in benign tumors (Table II), indicating the critical role of p53 in breast cancer as reported by previous studies $(10,11)$.

p53 mutation in breast tumors. To elucidate the mechanism underlying p53 accumulation in our breast tumor samples, we examined $p 53$ mutations in all tumor samples by DNA sequencing of the exons from 4 to 9 . No mutation was detected from exons 5 to 9 in all samples. A missense mutation in exon 4 was found in 1 out of 98 tumor samples (Table III). A to $\mathrm{G}$ point mutation (Val $\rightarrow \mathrm{Ala}$ ) at codon 103 in exon 4 was characterized. Numerous p53 mutations block the MDM2-mediated p53 degradation. However, no p53 mutation was detected in almost all our breast tumors, suggesting that the increase of p53 is not due to gene mutation as previously observed $(11,12)$. Therefore, other E3 ligases may be responsible for p53 degradation in these tumors.

Downregulation of TRAF7 expression in breast cancer. To search for other possible E3 ligases for p53, the mRNA expression of E3 ligases was examined using qRT-PCR. We found the E3 ligase TRAF7 mRNA level was largely reduced

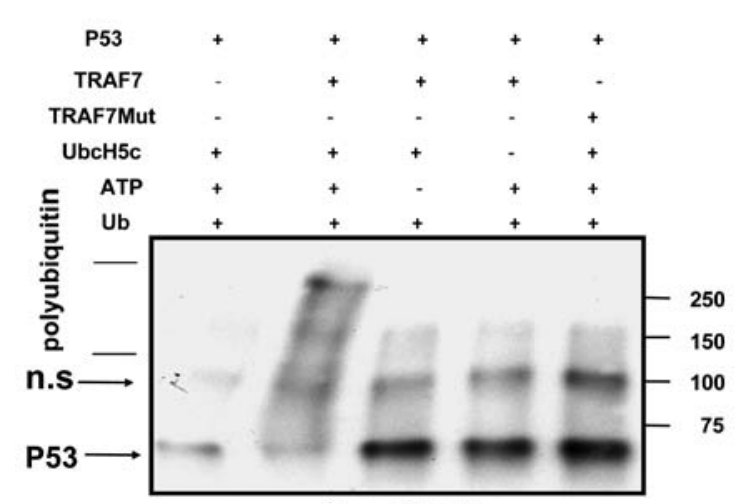

IB:anti-P53

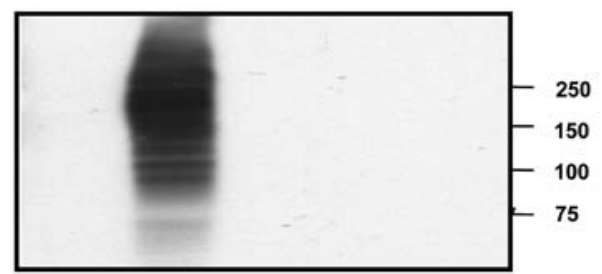

IP: anti-P53 IB:anti-Ub

Figure 2. TRAF7-mediated ubiquitination on $\mathrm{p} 53$ in vitro. In vitro ubiquitination reaction of p53 in the presence of TRAF7, or TRAF7 mutant (RING domain deletion). The reaction mixtures were immunoblotted using the indicated antibodies.

in breast tumors compared to the corresponding adjacent tissues $(\mathrm{P}<0.05)$ (Fig. 3A). TRAF7 protein was also markedly reduced in tumor specimens compared to adjacent tissues (Fig. 3B and C). The correlation between the reduction of TRAF7 and the increase of p53 suggests that TRAF7 may be an E3 ligase targeting p53 degradation.

TRAF7-mediated ubiquitination of p53. TRAF family members play a central role in the regulation of the TNF signaling pathway, especially in modulating the activation of NF- $\kappa B$ (13). Similar to the other TRAF family member, the RING domain of TRAF7 determines its function of E3 ubiquitin ligase (14). To test whether p53 was a potential target of TRAF7 for ubiquitination, we performed the in vitro ubiquitination assay. As shown in Fig. 2, p53 underwent K48-linked polyubiquitination in the presence of TRAF7. By contrast, no ubiquitinated p53 was detected when a TRAF7 mutant without the RING domain was added. These data suggest that TRAF7 polyubiquitinates $\mathrm{p} 53$ through the RING domain, which leads to 533 degradation.

Correlation between TRAF7 downregulation and poor prognosis. To validate the role of TRAF7 on p53, a public breast cancer cohort was used to evaluate the effect of TRAF7 expression on the survival of breast cancer patients using the Kaplan-Meier survival curves. As shown in Fig. 4A, a poorer prognosis was predicted in patients with low TRAF7 expression than in patients with high $T R A F 7$ expression. Moreover, patients with low TRAF7 and high $p 53$ expression (TRAF $77^{\text {low }} p 53^{\text {high }}$ ) showed a significant lower survival rate than patients with high TRAF7 and low $p 53$ expression (TRAF $7^{\text {high }} p 53^{\text {low }}$ (Fig. 4B). These observations suggest the critical role of TRAF7 on the prognosis of breast cancer by regulating p53 degradation. 


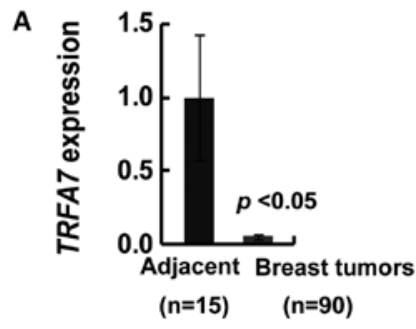

B

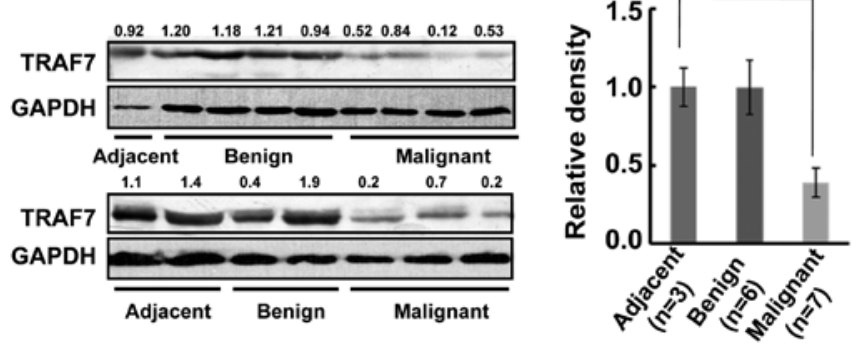

Figure 3. Reduced TRAF7 expression in breast cancer. (A) TRAF7 mRNA expression in breast tumors $(\mathrm{n}=95)$ compared to adjacent tissues $(\mathrm{n}=15)$ reflected by qRT-PCR analysis. (B) TRAF7 protein content in adjacent tissues and breast tumors shown by western blot analysis. (C) Quantified data of relative density of TRAF7 protein levels in adjacent tissues and breast tumors.
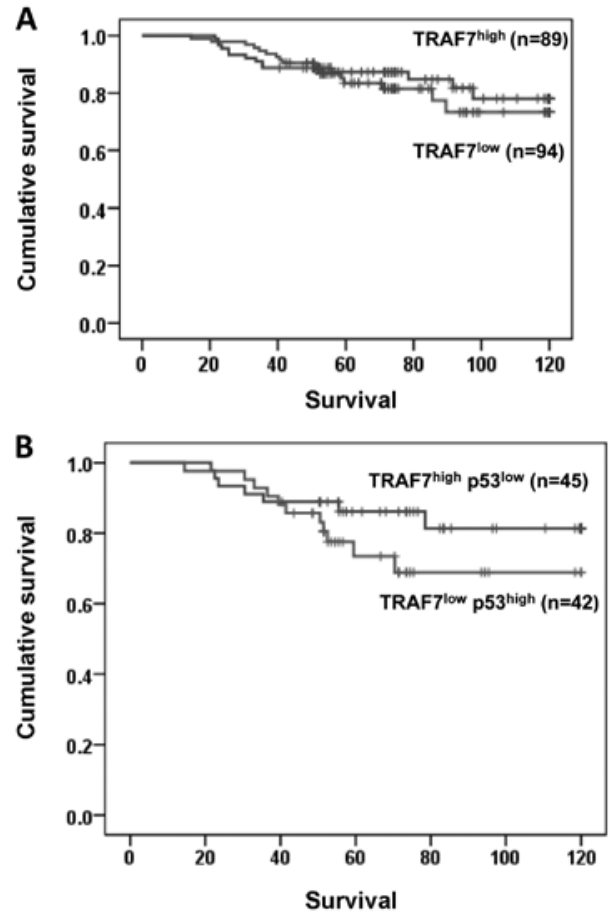

Figure 4. TRAF7 downregulation correlated with poor prognosis in a breast cancer cohort. The Kaplan-Meier survival curves were developed to depict the relationship between TRAF7 and/or $p 53 \mathrm{mRNA}$ expression and survival rates in a publicly available breast cancer cohort. (A) The survival curves related to TRAF7 mRNA level. (B) The survival curve describing the synergistic downregulation and upregulation of TRAF7 and $p 53$ mRNA expression.

\section{Discussion}

Ubiquitin-mediated degradation is the only way in which p53 is terminated by proteasomes (15). A few E3 ligases of p53 have been discovered thus far, of which MDM2 is the most cardinal one (16). In normal cells, a low level of p53 is maintained by its rapid degradation in a ubiquitin-dependent manner. Impairment of the p53 degradation pathway would cause its abnormal retention. Additionally, p53's functional deficiency might also interpret its accumulation in wt without involving structural mutations within the $p 53$ gene itself, such as cytosolic retention of p53 and its binding with blocking proteins $(17,18)$. Thus, increased wt p53 level in cancer suggests that the p53 functional signaling and/or degradation signaling is damaged to a certain degree, leading to defects in p53 protein degradation and deficiencies in its normal functions, which however is compensated and tolerated in cancer cells.

The accumulation of wt $\mathrm{p} 53$ has also been found in breast cancer $(11,12,15)$. However, the mechanism that leads to p53 accumulation is not yet well understood. In this study, we demonstrated that the E3 ligase TRAF7 could target p53 and increase its ubiquitination, suggesting a critical role of TRAF7 in regulating p53's protein stability. We observed p53 accumulation and corresponding TRAF7 downregulation in breast tumors, indicating the correlation between TRAF7 deregulation and p53 stabilization. Moreover, p53 accumulation was found to be prominently localized in cytoplasm, suggesting remarkable deficiencies of p53 functions in these tumors. Additionally, TRAF7 downregulation was verified to predict poor prognosis in breast cancer patients, particularly with a concomitant of $p 53$ upregulation. The combined data signified the importance of the TRAF7/p53 axis in modulating breast cancer development. Similar to the results from the current study, previous studies have also revealed that ungoverned p53 even in wt could significantly enhance tumor initiation and progression due to deregulation of the p53 network such as a fundamental switch of the expression pattern of its target genes $(19,20)$. For example, defects in MDM2's binding to p53 resulted in promoting wt p53 accumulation, leading to enhanced tumor progression in non-small cell lung cancer (21).

To the best of our knowledge, we are the first to find that TRAF7 is an E3 ligase targeted for p53 degradation. Meanwhile, we demonstrated that dramatic downregulation of TRAF7 expression in breast tumor specimens correlated with high levels of wt p53 protein in these tumors. Collectively, these results indicated that the accumulation of wt p53 in tumors was due to defects in the process of p 53 degradation, and TRAF7 downregulation largely contributed to provoke p53 cytosolic retention and its functional deficiencies. Therefore, the deregulated TRAF7/p53 axis presumably plays a crucial role in breast cancer development, and strategies targeting this axis might represent a novel approach to restrain tumor progression in breast cancer.

\section{Acknowledgements}

This study was supported by grants from the Chinese Academy of Sciences (KZCX2-EW-404), the National Natural Science Foundation of China (grant nos. 21077128, 20921063, 21177151) and from the program of 'Hundreds Talents' of the Chinese Academy of Sciences. We thank the lab members for their significant assistance with experiments and reagents. 


\section{References}

1. Hollstein M, Sidransky D, Vogelstein B and Harris C: p53 mutations in human cancers. Science 253: 49-53, 1991.

2. Scudiero I, Zotti T, Ferravante A, et al: Tumor necrosis factor (TNF) receptor-associated factor 7 is required for $\mathrm{TNF} \alpha$-induced Jun NH2-terminal kinase activation and promotes cell death by regulating polyubiquitination and lysosomal degradation of c-FLIP protein. J Biol Chem 287: 6053-6061, 2012.

3. Zotti T, Uva A, Ferravante A, et al: TRAF7 protein promotes Lys-29-linked polyubiquitination of IкB kinase $(\mathrm{IKK} \gamma) / \mathrm{NF}-\kappa \mathrm{B}$ essential modulator (NEMO) and p65/RelA protein and represses NF- $\kappa$ B activation. J Biol Chem 286: 22924-22933, 2011.

4. Tsikitis M, Acosta-Alvear D, Blais A, et al: Traf7, a MyoD1 transcriptional target, regulates nuclear factor- $\mathrm{\kappa B}$ activity during myogenesis. EMBO Rep 11: 969-976, 2010.

5. Patocs A, Zhang L, Xu Y, et al: Breast-cancer stromal cells with TP53 mutations and nodal metastases. N Engl J Med 357: 2543-2551, 2007

6. Kusser WC, Levin DB and Glickman BW: Sensitive two-stage PCR of p53 genomic DNA exons 5-9. PCR Methods Appl 2: 250-252, 1993 .

7. Liu S, Goldstein RH, Scepansky EM and Rosenblatt M: Inhibition of rho-associated kinase signaling prevents breast cancer metastasis to human bone. Cancer Res 69: 8742-8751, 2009

8. Liu S, Li S and Du Y: Polychlorinated biphenyls (PCBs) enhance metastatic properties of breast cancer cells by activating Rho-associated kinase (ROCK). PLoS One 5: e11272, 2010.

9. Jamshidi M, Bartkova J, Greco D, et al: NQO1 expression correlates inversely with $\mathrm{NF} \kappa \mathrm{B}$ activation in human breast cancer. Breast Cancer Res Treat 132: 955-968, 2012.

10. Silvestrini R, Daidone MG, Benini E, et al: Validation of p53 accumulation as a predictor of distant metastasis at 10 years of follow-up in 1400 node-negative breast cancers. Clin Cancer Res 2: 2007-2013, 1996.
11. Sjögren S, Inganäs M, Norberg T, et al: The p53 gene in breast cancer: prognostic value of complementary DNA sequencing versus immunohistochemistry. J Natl Cancer Inst 88: 173-182, 1996.

12. Moll UM, Riou G and Levine AJ: Two distinct mechanisms alter p53 in breast cancer: mutation and nuclear exclusion. Proc Natl Acad Sci USA 89: 7262-7266, 1992.

13. Häcker H, Tseng PH and Karin M: Expanding TRAF function: TRAF3 as a tri-faced immune regulator. Nat Rev Immunol 11: 457-468, 2011.

14. Zotti T, Vito P and Stilo R: The seventh ring: exploring TRAF7 functions. J Cell Physiol 227: 1280-1284, 2012.

15. Lacroix M, Toillon RA and Leclercq G: p53 and breast cancer, an update. Endocr Relat Cancer 13: 293-325, 2006.

16. Lee JT and Gu W: The multiple levels of regulation by p53 ubiquitination. Cell Death Differ 17: 86-92, 2009.

17. O'Brate A and Giannakakou P: The importance of p53 location: nuclear or cytoplasmic zip code? Drug Resist Updat 6: 313-322, 2003.

18. Liang SH and Clarke MF: Regulation of p53 localization. Eur J Biochem 268: 2779-2783, 2001.

19. Mullany LK, Liu Z, King ER, Wong KK and Richards JS: Wild-type tumor repressor protein 53 (Trp53) promotes ovarian cancer cell survival. Endocrinology 153: 1638-1648, 2012.

20. Houben R, Hesbacher S, Schmid CP, et al: High-level expression of wild-type p53 in melanoma cells is frequently associated with inactivity in p53 reporter gene assays. PLoS One 6: e22096, 2011.

21. Wang YC, Lin RK, Tan YH, Chen JT, Chen CY and Wang YC: Wild-type p53 overexpression and its correlation with MDM2 and p14ARF alterations: an alternative pathway to non-small cell lung cancer. J Clin Oncol 23: 154-164, 2005. 